\title{
Visual and topographic impacts of trans-epithelial versus epithelium-off corneal collagen cross-linking in adult keratoconus
}

\author{
Amani E. Badawi
}

Correspondence: amanibadawi37@yahoo.com

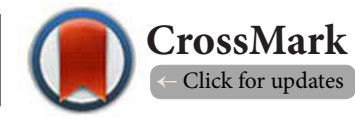

Department of Ophthalmology, Mansoura Ophthalmic Center, Faculty of Medicine, Mansoura University, Egypt.

\begin{abstract}
Purpose: To evaluate the efficacy of trans-epithelial cross-linking (TE-CXL) and to compare the visual and topographic outcomes of TE-CXL versus epithelium-off cross-linking (CXL) in adult keratoconus (KC).

Design: A prospective non-randomized open case series.

Methods: The study enrolled $27 \mathrm{KC}$ eyes of 15 patients treated by TE-CXL (group I) and $35 \mathrm{KC}$ eyes of 20 patients treated by epithelial-off CXL (group II). In group I, riboflavin 0.1\% solution in 15\% Dextran T500 with sodium EDTA $0.01 \%$ and trometamol (Ricrolin TE) was used with intact corneal epithelium. In group II, the epithelium was removed. Then, riboflavin $0.1 \%$ solution $(10 \mathrm{mg}$ of riboflavin 5 -phosphate in a $20 \%$ dextran T-500 10-mL solution (Ricrolin) was applied. Ultraviolet, A irradiation, was occurred using UVA system (CBM X-linker, at $\left.3 \mathrm{~mW} / \mathrm{cm}^{2}\right)$. Preoperative and postoperative assessment included uncorrected visual acuity (UCVA), best-corrected visual acuity (BCVA), corneal astigmatism, maximum keratometry (K max), central corneal thickness (CCT), thinnest corneal thickness, and spherical aberration using Wavelight oculyzer -pentacam HR. All included patients had at least one-year follow-up.
\end{abstract}

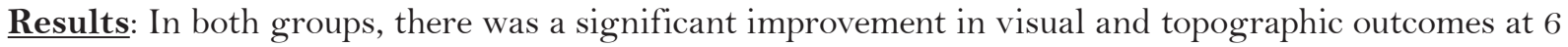
months postoperative. Furthermore, in theTE-CXL group; the vision and keratometric readings gradually deteriorated at the end of one year follow-up. Comparison of postoperative changes at 12 months between two groups demonstrated the epithelial-off CXL group had statistically better results concerning UCVA, BCVA, corneal astigmatism, and $\mathrm{K} \max (\mathrm{p}=0.03,0.002,0.001$, and $<0.001$ respectively).

Conclusions: Functional and clinical regression in TE-CXL group to the preoperative status after an initial improvement could conclude that TE-CXL cannot stabilize $\mathrm{KC}$ for a long term. Conventional CXL is superior on TE-CXL in KC stability after 12 months of follow-up.

Keywords: Keratoconus, cross-linking, epithelial-off, trans-epithelial, ricrolin-TE

\section{Introduction}

Corneal collagen cross-linking (CXL) has been known as a promising treatment for keratoconus (KC) and other ectasias. According to standard CXL protocol, epithelium should be removed before irradiation to allow good penetration of riboflavin into the corneal stroma [1,2]. However, de-epithelization may increase the risks rate as corneal infections, scarring, infiltrates and haze [3] Epithelial-on CXL (trans-epithelial) is a modified procedure recently introduced to reduce postop- erative pain and risk of infection [4]. Riboflavin cannot easily pass through tight junctions of the intact epithelium as it is a hydrophilic solution [5]. Epithelial permeability can be increased by topical drugs, such as ethylenediamine tetra-acetic acid (EDTA) and benzalkonium chloride $[6,7]$. In order to evaluate the efficacy of trans-epithelial cross-linking (TE-CXL) and to compare the visual and topographic outcomes of TE-CXL versus epithelium-off CXL in adult KC at the end of one year followup, this study was designed using a modified photosensitizer 
$0.1 \%$ riboflavin-15\% dextran solution supplemented with Tris-hydroxymethylaminomethane and sodium ethylenediaminetetraacetic acid versus the standard riboflavin solution.

\section{Materials and methods}

This is a prospective non-randomized open study of patients diagnosed as progressive $\mathrm{KC}$ during the period from February 2013 to March 2014. Progression was documented as an increase in $\mathrm{K}$ max of 1.00 diopter or more in the last 6 months with reported worsening of visual acuity of more than one Snellen line over previous 3-6 months. The inclusion criteria were stages I-III KC, according to the Krumeich classification, with a completely clear cornea. All patients included in the study were of age greater than 18 years. Thinnest corneal thickness was $\geq 370 \mu \mathrm{m}$. Based on the thinnest pachymetry values, the conducted patients were divided into two groups. Group I (370-400 $\mu \mathrm{m}$ )-whom the standard CXL cannot be applied-was subjected to TE- CXL and group II (more than 400) was treated with standard CXL.

Exclusion criteria were any previous ocular surgery, corneal opacities, history of herpetic keratitis or active ocular infection, pregnancy, lactation, and severe dry eye.

All applicable institutional and governmental regulations concerning the ethical use of human participants were followed during this study according to the principles of the Declaration of Helsinki, and the local ethics committee of Mansoura University approved the study IRB: 16.08.20. All patients signed an informed consent form.

\section{Preoperative evaluation}

All patients in both groups underwent a complete ophthalmologic examination, including uncorrected visual acuity (UCVA), best-corrected visual acuity (BCVA) assessment, slit lamp biomicroscopy, fundus examination using indirect ophthalmoscopy and non- contact Volk 90 lens, corneal astigmatism, and corneal tomography with measured pachymetry by (Wavelight oculyzer - pentacam HR; WaveLight GmbH, Germany).

\section{Surgical technique}

The procedure was conducted under sterile conditions in the operating room of Al Hayah Hospital for Eye Surgery\&LASIK at Mansoura-Egypt. In Group I, corneal epithelium was left intact. Riboflavin $0.1 \%$ solution in $15 \%$ Dextran T500 with sodium EDTA $0.01 \%$ and trometamol (Ricrolin TE ${ }^{\circledR}$, Sooft, Montegiorgio, Italy) was instilled as two drops every 5 minutes for 30 minutes till complete saturation of the stroma. Before UV radiation, topical anesthetic eye drop was applied to the cornea with benoxinate hydrochloride $0.4 \%$ (Benox $4 \%$; Eipico Inc., Cairo, Egypt) every 5 minutes for 20 minutes. Ultraviolet, A irradiation, was occurred using a commercially available UVA system (CBM X-linker, CSO, Florence, Italy). Before treatment, the intended $3 \mathrm{~mW} / \mathrm{cm}^{2}$ surface irradiance $\left(5.4 \mathrm{~J} / \mathrm{cm}^{2}\right.$ surface dosages after 30 minutes) was calibrated. During treatment, riboflavin solution was applied every 2 minutes to ensure saturation. At the end of the procedure, the eye was washed well with balanced salt solution.

In Group II, the epithelium was mechanically removed with surgical Beaver blade within the central $8 \mathrm{~mm}$ diameter. Then, riboflavin $0.1 \%$ solution $(10 \mathrm{mg}$ of riboflavin 5 -phosphate in a $20 \%$ dextran T-500 10-mL solution (Ricrolin ${ }^{\circledR}$, Sooft, Montegiorgio, Italy) was applied to the cornea every 3 minutes for 30 minutes to achieve adequate penetration of the solution. Ultraviolet- A irradiation was applied by the same protocol for CXL as in group I. Contact lens was prescribed to Group II and then removed after complete epithelial healing.

\section{Postoperative care}

Patients received moxifloxacin hydrochloride $0.5 \%$ (Vigamox ${ }^{\circledR}$; Alcon Inc., Texas, USA) and nepafenac $0.1 \%$ ophthalmic suspension (Nevanac ${ }^{\circledR}$; Alcon Inc., Texas, USA) 4 times daily for one week. Then, topical steroid-antibiotic drops (Tobradex ${ }^{\circledR}$; Alcon Inc., Texas, USA) 4 times daily for a week which tapered over the next 3 weeks to zero; and, Carboxymethylcellulose Sodium (CMC) $(0.5 \%)$ eye lubricant (Refresh Tears ${ }^{\circledR}$; Allergan, Inc.,California, USA.) 6 times daily for one month.

Follow-up was done after one day then after one week for prescription of steroid-antibiotic drops and one month for assessment of corneal haze. Uncorrected visual acuity (UCVA), $\mathrm{BCVA}$, corneal astigmatism, $\mathrm{K}$ max, central corneal thickness (CCT), thinnest corneal thickness, and spherical aberration were recorded at 6,12 months in both groups.

\section{Data analysis}

Snellen's visual acuity was changed into logarithm of the Minimum Angle of Resolution (logMAR) as recommended by Holladay [8] for further statistical analysis. The raw data was collected on excel sheets and imported to the Statistical Package for Social Sciences (SPSS Inc., Chicago, IL, version $15.0)$ to get the mean and standard deviation. Paired $t$-test was used to compare preoperative and postoperative data. The difference between the values of the two groups at baseline and postoperative was assessed using unpaired two sample Student $t$-test. The significance level was set at $\mathrm{P}<0.05$.

\section{Results}

Group I included 27 eyes of 15 patients; 12 patients (bilateral eyes) and 3 patients (unilateral eyes). The mean age of patients was $22.21 \pm 2.75$ years (range 18 to 25 years). Ten patients (66.67\%) were females and 5 (33.33\%) were males. Group II included 35 eyes of 20 patients; the mean age of patients was $23.45 \pm 2.13$ years (range 19 to 27 years). Twenty patients $(60 \%)$ were females and $8(40 \%)$ were males. Group I showed a statistically significant improvement from the preoperative values $(P<0.001)$. The mean baseline UCVA was $0.82 \pm 0.04$ which improved to $0.48 \pm 0.20$ at 6 months and changed (worsened) to $0.68 \pm 0.03$ at one year with $(P<0.001)$. In group II the same improvement was recorded. The mean baseline UCVA changed from $0.80 \pm 0.04$ to $0.46 \pm 0.20,0.45 \pm 0.03$ at 6 
months and 12 months respectively.

The preoperative mean BCVA in group I was $0.57 \pm 0.03$ and changed to $0.38 \pm 0.02$ at 6 months and $0.48 \pm 0.02$ at one year. In spite, there was a statistically significant $(P<0.001)$ improvement in BCVA between the preoperative and 6, 12 months; the mean BCVA worsened from 6 months to 12 months. The improvement was starting from the first month to the sixth month postoperatively and then declined from 6 to 12 months. Seven eyes (25.93\%) gained one or two lines in BCVA, eleven eyes $(40.74 \%)$ maintained the preoperative BCVA; and nine eyes (33.33\%) lost one or two lines of the preoperative BCVA. While in group II the BCVA improvement continued until the end of follow- up period. It improved from $0.47 \pm 0.03$ to $0.32 \pm 0.02$ at 6 months and to $0.30 \pm 0.02$ at one year. Figure 1 shows the changes in BCVA through one year in both groups.

The median baseline anterior $\mathrm{K}$ max value in group I was $48.34 \pm 0.45 \mathrm{D}$ and improved significantly to $46.18 \pm 0.45(\mathrm{P}<0.001)$ at 6 months then, unfortunately, changed to $47.98 \pm 0.32(P>0.001)$ at one year with no significant differences from preoperative values. Progression of corneal steepening was observed in 10 eyes $(37.04 \%)$ of the treated eyes, 8 eyes $(29.62 \%)$ maintained the preoperative value and only 5 eyes (18.51\%) showed improvement. Contrary in group II, no disease progression was reported during the follow-up period. The mean anterior $\mathrm{K}$ max significantly changed from $48.14 \pm 0.45 \mathrm{D}$ preoperatively to $45.4 \pm 0.32 \mathrm{D}$ at 12 months $(P<0.001)$. Figure 2 shows the changes in $\mathrm{K}$ max through one year in both groups.

The mean corneal astigmatism values showed statistically significant changes in both groups. In the TE-CXL group, its values at 6 month and 1-year post treatment were statistically significant (mean $3.89 \pm 0.31 \mathrm{D}$ and $4.68 \pm 0.25 \mathrm{D}$ respectively) less than the preoperative values (mean 5.02 $\pm 0.25 \mathrm{D}$ ). Similarly, in standard CXL group, it significantly changed from $4.01 \pm 0.25$ $D$ to $3.21 \pm 0.25 \mathrm{D}$ at one year. There was no statistically significant correlation between corneal astigmatism and visual

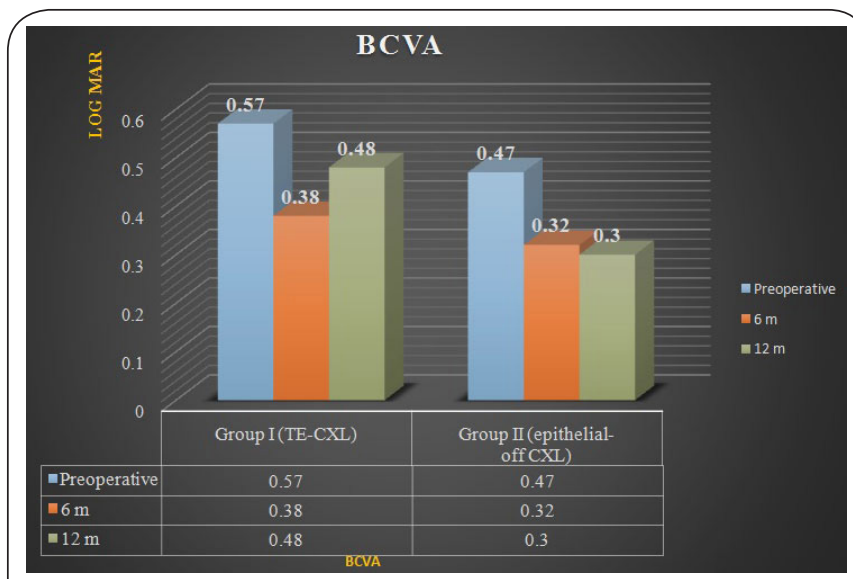

Figure 1. Comparison between the preoperative and postoperative mean BCVA among studied groups. CXL: Cross-linking; TE-CXL: Trans-epithelial cross-linking; BCVA: Best corrected visual acuity.

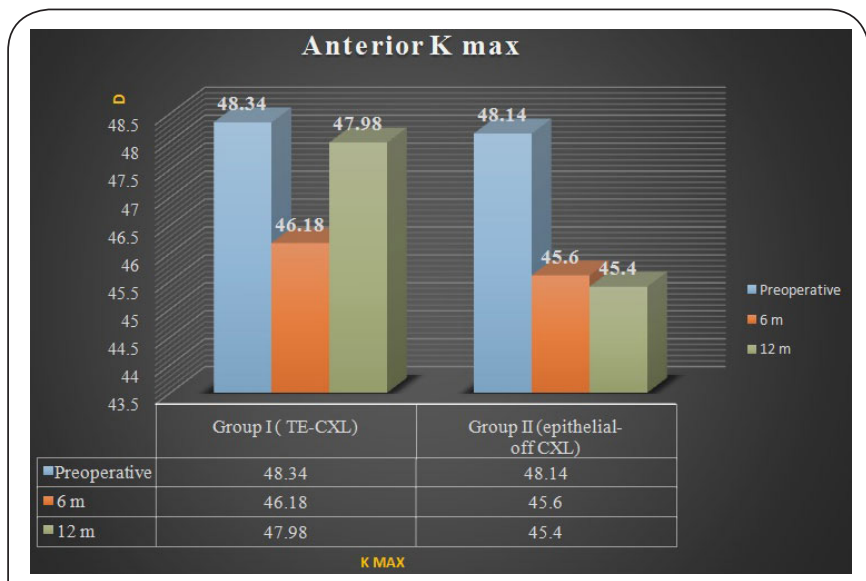

Figure 2. Comparison between the preoperative and postoperative mean anterior $\mathrm{K}$ max among studied groups. CXL: Cross-linking; TE-CXL: Trans-epithelial cross-linking; D: Diopter; K max: Maximum keratometric value.

outcomes in both groups. In group I, the mean baseline CCT was $419.91 \pm 6.66 \mu \mathrm{m}$. At 6 months and 12 months, the values reduced to $405.1 \pm 5.02 \mu \mathrm{m}$ and $401.24 \pm 5.31 \mu \mathrm{m}$ respectively. There was a statistically significant difference between the baseline and 12 months $(P<0.001)$ after the procedure.Similarly, the mean thinnest location was $389.91 \pm 6.66 \mu \mathrm{m}$ at baseline, $374.1 \pm 5.02 \mu \mathrm{m}$ at 6 months and the end of 12 months it was $370.24 \pm 5.31 \mu \mathrm{m}$. Mean aberration coefficient (spherical aberration) reduced from $1.07 \pm 0.23$ preoperatively to $0.72 \pm 0.20$ at 6 months and changed to $0.97 \pm 0.23$ at 12 months postoperatively with $(P>0.001)$. On the other hand, the epithelial-off group showed different results. The mean preoperative CCT was $463.44 \pm 6.19 \mu \mathrm{m}$ which changed to $447.68 \pm 5.38$ at 6 months and to $444.25 \pm 5.19$ at 12 months. There was a statistically significant difference between the baseline and 12 months $(P<0.001)$ after the procedure. The mean thinnest location was $439.91 \pm 6.66 \mu \mathrm{m}$ at baseline, and reduced to $425.1 \pm 5.02$ um at 6 months and the end of 12 months it was $420.24 \pm 5.31$ um. Mean aberration coefficient (spherical aberration) in this group showed improvement from $0.84 \pm 0.23$ preoperatively to $0.62 \pm 0.22$ at 6 months and changed to $0.63 \pm 0.23$ at 12 months postoperatively with $(P<0.001)$. No major complications were noted in this series, except for postoperative mild corneal haze with little impact on BCVA. The study results showed stabilization and improvement in adult $\mathrm{KC}$ in terms of VA and corneal $\mathrm{K}$ max after TE-CXL for a short period only and the vision and keratometric readings gradually worsened at the end of one year follow up with a negligible difference from preoperative values. For more detailed data, (Tables 1 and 2) show the mean baseline and postoperative at $6 \& 12$ months of the studied parameters (UCVA, BCVA corneal astigmatism, $\mathrm{K}$ max, pachymetry and spherical aberration) with the statistical significance of the changes ( $P$ values) in group I\&ll respectively.

The postoperative visual and the topographic changes were evaluated and differences between the two groups were 
Amani E. Badawi, Journal of Eye and Ophthalmology 2016,

http://www.hoajonline.com/journals/pdf/2055-2408-3-1.pdf

doi: $10.7243 / 2055-2408-3-1$

Table 1. The mean preoperative and postoperative parameters and its $P$ values in group I (TE-CXL).

\begin{tabular}{llllll}
\hline Studied parameters & $\begin{array}{l}\text { Baseline Mean } \pm S D \\
\text { Min-max }\end{array}$ & $\begin{array}{l}\mathbf{6} \text { m Mean } \pm S D \\
\text { Min-max }\end{array}$ & $\boldsymbol{P}^{I}$-Value & $\begin{array}{l}\mathbf{1 2} \mathbf{m} \text { Mean } \pm S D \\
\text { Min-max }\end{array}$ & $\boldsymbol{P}^{2}$-value \\
\hline UCVA & $0.82 \pm 0.04(0.4-1.00)$ & $0.48 \pm 0.20(0-0.8)$ & $<0.001^{*}$ & $0.68 \pm 0.03(0.2-1.00)$ & $<0.001^{*}$ \\
BCVA & $0.57 \pm 0.03(0-0.8)$ & $0.38 \pm 0.02(0-0.5)$ & $<0.001^{\star}$ & $0.48 \pm 0.02(0-0.6)$ & $<0.001^{\star}$ \\
Corneal Astigmatism & $5.02 \pm 0.25(2.3-6.3)$ & $3.89 \pm 0.31(1.4-5.2)$ & $<0.001^{\star}$ & $4.68 \pm 0.25(2.4-5.94)$ & $<0.001^{\star}$ \\
K max (D) & $48.34 \pm 0.45(43.5-58.35)$ & $46.18 \pm 0.45(41.6-56.15)$ & $<0.001^{*}$ & $47.98 \pm 0.32(42.6-57.14)$ & $>0.001$ \\
CCT & $419.91 \pm 6.66(384-435)$ & $405.1 \pm 5.02(368-410)$ & $<0.001^{*}$ & $401.24 \pm 5.31(366-415)$ & $<0.001^{*}$ \\
Thinnest location & $389.91 \pm 6.66(375-398)$ & $374.1 \pm 5.02(354-376)$ & $<0.001^{*}$ & $370.24 \pm 5.31(351-372)$ & $<0.001^{*}$ \\
spherical aberration & $1.07 \pm 0.23(0.98-1.2)$ & $0.72 \pm 0.20(0.6-1.01)$ & $<0.001^{*}$ & $0.97 \pm 0.23(0.8-1.06)$ & $>0.001$ \\
\hline
\end{tabular}

Test used: Paired -t-test

"“” Statistically significant differences when $\mathrm{p}<0.05$.

P1: difference between preoperative and after 6month; P2: difference between preoperative and 12 month.

CXL: Cross-linking; TE-CXL: Trans-epithelial cross-linking; D: Diopter ;SD: Standard deviation; UCVA: Uncorrected visual acuity; BCVA: Best corrected visual acuity; CCT: Central corneal thickness; K max: Maximum keratometric value.

Table 2. The mean preoperative and postoperative parameters and its $P$ values in group II(epithelium-off CXL).

\begin{tabular}{|c|c|c|c|c|c|}
\hline Studied parameters & $\begin{array}{l}\text { Baseline Mean } \pm S D \\
\text { Min-max }\end{array}$ & $\begin{array}{l}6 \text { m Mean } \pm S D \\
\text { Min-max }\end{array}$ & $P^{I}$-Value & $\begin{array}{l}12 \mathrm{~m} \operatorname{Mean} \pm S D \\
\text { Min-max }\end{array}$ & $P^{2}$-value \\
\hline UCVA & $0.80 \pm 0.04(0.5-1.00)$ & $0.46 \pm 0.20(0-0.7)$ & $<0.001^{\star}$ & $0.45 \pm 0.03(0-0.5)$ & $<0.001^{\star}$ \\
\hline BCVA & $0.47 \pm 0.03(0-0.7)$ & $0.32 \pm 0.02(0-0.3)$ & $<0.001^{\star}$ & $0.30 \pm 0.02(0-0.5)$ & $<0.001^{\star}$ \\
\hline Corneal Astigmatism & $4.01 \pm 0.25(1.3-5.2)$ & $3.22 \pm 0.31(0.8-4.4)$ & $<0.001^{\star}$ & $3.21 \pm 0.25(0.8-4.2)$ & $<0.001^{\star}$ \\
\hline $\mathrm{K} \max (\mathrm{D})$ & $48.14 \pm 0.45(43-57.5)$ & $45.6 \pm 0.45(41.6-54.2)$ & $<0.001^{\star}$ & $45.4 \pm 0.32(41.6-55.4)$ & $<0.001^{\star}$ \\
\hline CCT & $463.44 \pm 6.19(440-470)$ & $447.68 \pm 5.38(423-454)$ & $<0.001^{\star}$ & $444.25 \pm 5.19(422-455)$ & $<0.001^{\star}$ \\
\hline Thinnest location & $439.91 \pm 6.66(419-457)$ & $425.1 \pm 5.02(401-436)$ & $<0.001^{\star}$ & $420.24 \pm 5.31(398-437)$ & $<0.001^{\star}$ \\
\hline spherical aberration & $0.84 \pm 0.23(1.02-0.72)$ & $0.62 \pm 0.22(0.95-0.057)$ & $<0.001^{\star}$ & $0.63 \pm 0.23(0.94-0.58)$ & $<0.001^{\star}$ \\
\hline
\end{tabular}

Test used: Paired -t-test

"^” Statistically significant differences when $\mathrm{p}<0.05$

P1: difference between preoperative and after 6 month.

P2: difference between preoperative and 12 month.

CXL: Cross-linking; TE-CXL: Trans-epithelial cross-linking; D: Diopter; SD: Standard deviation; UCVA: Uncorrected visual acuity; BCVA: Best corrected visual acuity; CCT: Central corneal thickness; K max: Maximum keratometric value.

calculated. Table 3 shows the comparison of postoperative changes at 6,12 months between two groups. No statistically significant differences were observed between two groups of postoperative changes at 6 months. While, the epithelial-off CXL group showed statistically better results of postoperative changes at 12 months concerning UCVA, BCVA, corneal astigmatism, and $\mathrm{K} \max (\mathrm{p}=0.03,0.002,0.001$, and $<0.001$ respectively) Table 3.

\section{Discussion}

Trans-epithelial CXL, a modified technique of standard CXL, was introduced with the purpose to reduce the associated complications of corneal epithelial removal in traditional method $[4,9,10]$. Moreover, the procedure becomes more comfortable, less time consuming and more suitable for thin corneas [11]. Many studies were published with inconsistent results about the TE-CXL outcomes. Here the study reported a short-term clinical experience with TE-CXL in treating progressive $K C$ compared to the standard CXL results.
Clinically, there was a prominent improvement in the visual acuity in the current study in both groups. But this improvement was transient and fugacious in group I, making it difficult to formulate the efficacy of TE-CXL in disease stability. The same occurred in the other studied parameters which showed a marked improvement during the first six months but quickly deteriorated to reach close to the values before the operation. On the contrary, the improvement and disease stabilization continued for the end of the period of followup in the second group (epithelial -off CXL). Caporossi et al., reported convergent results in their study of trans-epithelial CXL included 26 eyes. They observed a temporary increase in both UCVA and BCVA within the first 3 months only. The final results were disappointing with progressive reduction and gradual return to preoperative values [12]. The topographic analysis in group I showed an initial statistically significant decrease in anterior $\mathrm{K}$ max within the first 6 months after the procedures then the collected data showed a worsening at the end of one year. Figure 3 shows an example of anterior $\mathrm{K}$ 
Amani E. Badawi, Journal of Eye and Ophthalmology 2016,

http://www.hoajonline.com/journals/pdf/2055-2408-3-1.pdf

doi: $10.7243 / 2055-2408-3-1$

Table 3. Comparison of post- CXL parameters between the two groups (TE-CXL andepithelium-off CXL).

\begin{tabular}{lllllll}
\hline Studied parameters & \multicolumn{3}{c}{ 6 months } & \multicolumn{3}{c}{ 12 months } \\
\hline & Group I & Group II & P-value & Group I & Group II & P-value \\
\hline UCVA & $0.34 \pm 0.02$ & $0.34 \pm 0.04$ & 0.98 & $0.14 \pm 0.04$ & $0.35 \pm 0.03$ & $0.03^{*}$ \\
BCVA & $0.19 \pm 0.03$ & $0.15 \pm 0.04$ & 0.48 & $0.09 \pm 0.03$ & $0.17 \pm 0.03$ & $0.002^{*}$ \\
Corneal Astigmatism & $0.34 \pm 0.25$ & $0.79 \pm 0.31$ & 0.34 & $0.13 \pm 0.31$ & $0.8 \pm 0.25$ & $0.001^{*}$ \\
K max (D) & $2.16 \pm 0.45$ & $2.54 \pm 0.45$ & 0.63 & $0.36 \pm 0.32$ & $2.74 \pm 0.32$ & $<0.001^{*}$ \\
CCT & $14.81 \pm 5.02$ & $15.76 \pm 5.38$ & 0.06 & $18.67 \pm 5.31$ & $19.19 \pm 5.19$ & 0.12 \\
Thinnest location & $15.81 \pm 5.02$ & $14.81 \pm 5.02$ & 0.64 & $19.67 \pm 5.31$ & $19.67 \pm 5.33$ & 0.97 \\
spherical aberration & $0.35 \pm 0.20$ & $0.22 \pm 0.22$ & 0.53 & $0.1 \pm 0.23$ & $0.21 \pm 0.23$ & 0.71 \\
\hline
\end{tabular}

Test used: Two sample t-tests

“»” Statistically significant differences when $\mathrm{p}<0.05$.

P1: difference between preoperative and after 6 month.

P2: difference between preoperative and 12 month.

CXL: Cross-linking; TE-CXL: Trans-epithelial cross-linking; D: Diopter ; SD: Standard deviation;

UCVA: Uncorrected visual acuity; BCVA: Best corrected visual acuity; CCT: Central corneal

thickness; K max: maximum keratometric value.

max changes after TE-CXL.

Changes in the pachymetry showed a statistically significant progressive thinning at 12 months in both groups with significant improvement of other topographic indices in epithelial -off group only. This significant reduction in corneal thickness should be put in mind respecting to the changes of other reported clinical results in the TE-CXL group. The study patients were categorized into each group based on corneal thickness depending on the safety standards outlined stated by Caporossi and his colleagues [13], the TE-CXL was applied to patients with a corneal thickness less than 400 in thinnest location. Some will find this bias may lead to the interpretation of study results. But, in spite of the better results of postoperative changes of the epithelia-off group at 12 months concerning UCVA, BCVA, corneal astigmatism, and $\mathrm{K}$ max; there was no an observed statistically significant difference between two groups of postoperative $\mathrm{CT}$ changes. It seems that there are other parameters rather than the corneal thickness would be responsible for the post-CXL changes. Furthermore, it is still till now unclear to predict the post-CXL results and which patients are more probable to benefit from the treatment. In clinical practice, few studies evaluated different preoperative parameters effects on post-CXL outcomes. Regarding the preoperative thinnest corneal pachymetry and its effect on CXL- outcomes, it was concluded that a thinnest corneal thickness less than $450 \mu \mathrm{m}$ significantly lead to more improving and flattening in the maximum $\mathrm{K}$ [14]. The preoperative pachymetry effect on CXL-outcomes is considered an interesting and controversial point; therefore it will need to be evaluated in a randomized study with a large sample size in the future.

Trans-epithelial CXL group shows a statistically significant variance in spherical aberration in the first 6 months, which partially explained the former tendency of functional improvement. However, it turned back closer to baseline values at the 12 months. While in group II, there was a statistically significant improvement in spherical aberration at 12 months.

Overall analysis of the current clinical outcomes after TECXL showed the disease stability was relatively temporary within the first 6 months in comparison with the conventional CXL results. Of 27 eyes, $29.62 \%$ maintained the preoperative level and only $18.51 \%$ showed clinical improvement at the end of follow-up.

Many published laboratory or clinical studies on this topic were comparable to our results and reported weaker or even no effect of TE-CXL in halt progression of ecstatic diseases [15-17]. In the present study about $37.04 \%$ of group I eyes showed a continued KC progression with a clinically significant deterioration concerning increased $\mathrm{K}$ max during the period from 6 months to 12 months follow up. Soeters et al., reached the same conclusion and reported $23 \%$ of cases progressed after one year [18].

Compared to the efficacy and the results of standard CXL, many investigators concluded that TE-CXL was significantly weaker and unstable and so hypothesized the cause due to minimal penetration of riboflavin, a hydrophilic molecule, through intact epithelium with resultant poor stromal saturation $[15,16]$. Currently, it was reported the efficacy of the epithelial -off $C X L$ in halt progression over TE-CXL and even statistically significant improvement in visual and topographic parameters It was found that the corneal biomechanical stiffening after TE-CXL about one-fifth compared to the standard (epithelium-off) CXL in an animal model [19]. Therefore TE- CXL was believed to be favorable procedure but with limited effects than standard CXL in treating and stabilizing progressive $\mathrm{KC}$ $[12,17,20]$. On the other, a study done by Filippello and his colleagues [4] reported better clinical and instrumental results with a statistically significant improvement in both visual and topographic parameters. They assumed that epithelial 


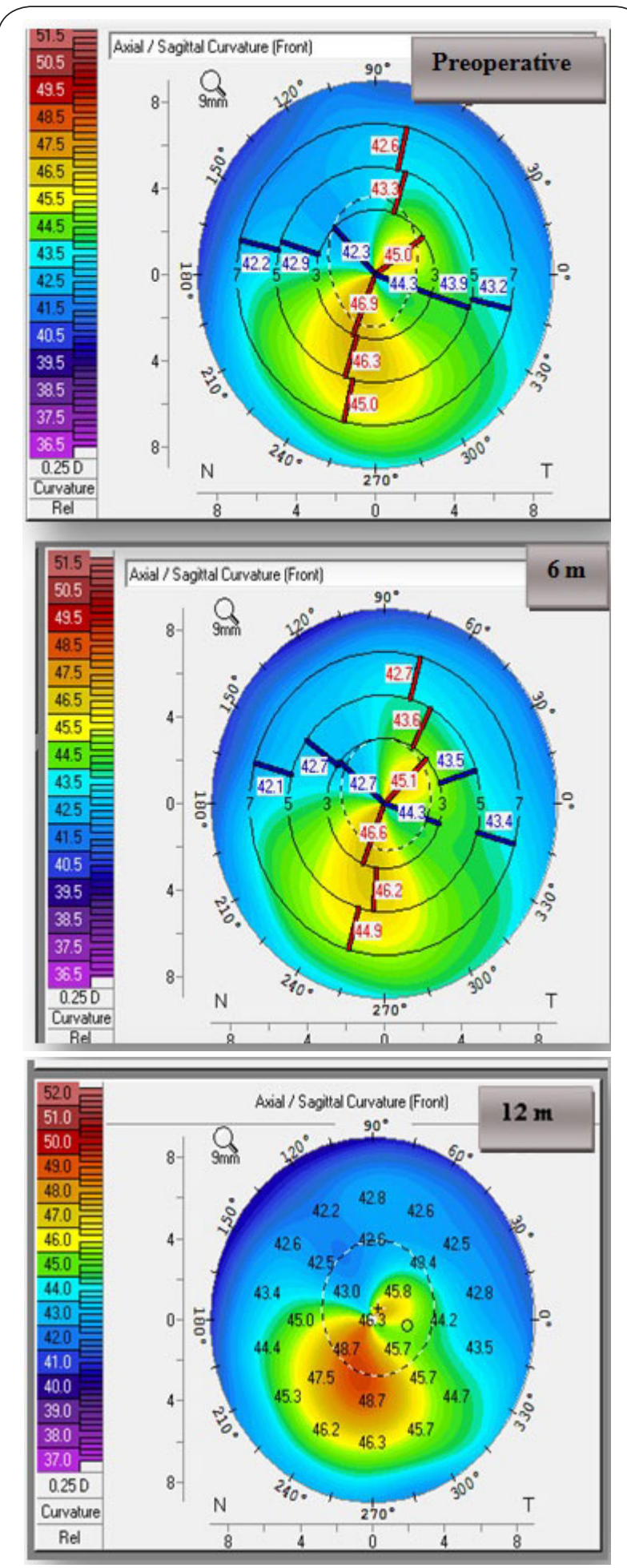

Figure 3. Pentacam (anterior curvature map) of female KC patient (22 years old) showed minimal improvement in anterior $\mathrm{K} \max$ from (46.9 D preoperatively) to $46.6 \mathrm{D}$ at 6 months with worsening to $48.7 \mathrm{D}$ at 12 months after TE-CXL.

KC: Keratoconus;TE-CXL: Trans-epithelial cross-linking; D: Diopter; K max: Maximum keratometric value. on CXL treatment was safe, noninvasive and well-tolerated.

Another retrospective study using modified protocol (mechanical disruption of the superficial epithelium, and an increase of the riboflavin-induction time), concluded the improvements in both visual, and topographic parameters after TE-CXL. Thus it appeared to be effective in treating progressive KC and to stop the progression [21].

Actually, several methods have been used to facilitate the riboflavin penetration through the intact epithelium, in addition, different inclusion criteria and different studies designs making comparison difficult. Currently, Ricrolin TE was used which known as the least hypotonic photosensitizer causing disruption effect of the corneal epithelium, with no reported epithelial defects and minimal postoperative pain. However, Ricrolin TE caused a shallow penetration of the riboflavin due to lack of some epithelial disruption which is necessary for perfect diffusion $[13,22]$. This may explain the limited unstable effects of TE-CXL in our results. What we can infer from this study, epithelial debridement affects the final clinical outcomes and conventional CXL is superior on TE-CXL in KC stability. However, these findings contradict the results of some researchers of the effectiveness of the TE-CXL $[4,11,21]$.

The limitations of the present study were the small number of the patients, non-randomized design and the short followup time. A large randomized controlled study with larger number of patients and longer follow-up is recommended to evaluate the long-term indications and outcomes of TE-CXL.

\section{Conclusion}

It could be concluded that one-year follow-up of the studied eyes was clear evidence of functional and clinical regression in the TE-CXL group to the preoperative status. This general return to the preoperative status in all parameters after an initial improvement proposes that TE- CXL cannot stabilize KC for a long term and does not outcome biomechanical stiffening needed for continuous clinical stability and improvement as standard epithelial on CXL.

\section{Competing interests}

The author declares that he has no competing interests.

\section{Acknowledgement}

All staff members in Mansoura Ophthalmic Center- Mansoura University and Al Hayah Hospital for Eye Surgery \& LASIK.

\section{Publication history}

Editor: Alparslan Sahin, Dicle University, Turkey.

Received: 03-Aug-2016 Final Revised: 08-Sep-2016

Accepted: 17-Sep-2016 Published: 29-Sep-2016

\section{References}

1. Wollensak G, Spoerl E and Seiler T. Riboflavin/ultraviolet-a-induced collagen crosslinking for the treatment of keratoconus. Am J Ophthalmol. 2003; 135:620-7. | Article | PubMed

2. Caporossi A, Baiocchi S, Mazzotta C, Traversi C and Caporossi T. 
Parasurgical therapy for keratoconus by riboflavin-ultraviolet type $A$ rays induced cross-linking of corneal collagen: preliminary refractive results in an Italian study. J Cataract Refract Surg. 2006; 32:837-45. I Article I PubMed

3. Kymionis GD, Bouzoukis DI, Diakonis VF, Portaliou DM, Pallikaris Al and Yoo SH. Diffuse lamellar keratitis after corneal crosslinking in a patient with post-laser in situ keratomileusis corneal ectasia. J Cataract Refract Surg. 2007; 33:2135-7. | Article | PubMed

4. Filippello M, Stagni E and O'Brart D. Transepithelial corneal collagen crosslinking: bilateral study. J Cataract Refract Surg. 2012; 38:283-91. I Article | PubMed

5. Spoerl E, Huhle M and Seiler T. Induction of cross-links in corneal tissue. Exp Eye Res. 1998; 66:97-103. | Article | PubMed

6. Chang SW, Chi RF, Wu CC and Su MJ. Benzalkonium chloride and gentamicin cause a leak in corneal epithelial cell membrane. Exp Eye Res. 2000; 71:3-10. | Article | PubMed

7. Nakamura T, Yamada M, Teshima M, Nakashima M, To H, Ichikawa $N$ and Sasaki H. Electrophysiological characterization of tight junctional pathway of rabbit cornea treated with ophthalmic ingredients. Biol Pharm Bull. 2007; 30:2360-4. | Article | PubMed

8. Holladay JT. Visual acuity measurements. J Cataract Refract Surg. 2004; 30:287-90. | Article | PubMed

9. Spoerl E, Hoyer A, Pillunat LE and Raiskup F. Corneal cross-linking and safety issues. Open Ophthalmol J. 2011; 5:14-6. | Article | PubMed Abstract | PubMed FullText

10. Hovakimyan M, Guthoff RF and Stachs O. Collagen cross-linking: current status and future directions. J Ophthalmol. 2012; 2012:406850. | Article | PubMed Abstract | PubMed FullText

11. Filippello M, Stagni E, Buccoliero D, Bonfiglio V and Avitabile T. Transepithelial cross-linking in keratoconus patients: confocal analysis. Optom Vis Sci. 2012; 89:e1-7. | Article | PubMed

12. Caporossi A, Mazzotta C, Paradiso AL, Baiocchi S, Marigliani D and Caporossi T. Transepithelial corneal collagen crosslinking for progressive keratoconus: 24-month clinical results. J Cataract Refract Surg. 2013; 39:1157-63. | Article | PubMed

13. Caporossi A, Mazzotta C, Baiocchi S, Caporossi T and Paradiso AL. Transepithelial corneal collagen crosslinking for keratoconus: qualitative investigation by in vivo HRT II confocal analysis. Eur J Ophthalmol. 2012; 22 Suppl 7:S81-8. | Article | PubMed

14. Toprak I, Yaylali V and Yildirim C. Factors affecting outcomes of corneal collagen crosslinking treatment. Eye (Lond). 2014; 28:41-6. | Article | PubMed Abstract | PubMed FullText

15. Samaras K, O'Brart D P, Doutch J, Hayes S, Marshall J and Meek KM. Effect of epithelial retention and removal on riboflavin absorption in porcine corneas. J Refract Surg. 2009; 25:771-5. | Article | PubMed

16. Hayes S, O'Brart DP, Lamdin LS, Doutch J, Samaras K, Marshall J and Meek KM. Effect of complete epithelial debridement before riboflavinultraviolet-A corneal collagen crosslinking therapy. J Cataract Refract Surg. 2008; 34:657-61. | Article | PubMed

17. Leccisotti $A$ and Islam T. Transepithelial corneal collagen cross-linking in keratoconus. J Refract Surg. 2010; 26:942-8. | Article | PubMed

18. Soeters N, Wisse RP, Godefrooij DA, Imhof SM and Tahzib NG. Transepithelial versus epithelium-off corneal cross-linking for the treatment of progressive keratoconus: a randomized controlled trial. Am J Ophthalmol. 2015; 159:821-8 e3. | Article | PubMed

19. Wollensak $G$ and lomdina E. Biomechanical and histological changes after corneal crosslinking with and without epithelial debridement. $J$ Cataract Refract Surg. 2009; 35:540-6. | Article | PubMed

20. Koppen C, Wouters K, Mathysen D, Rozema J and Tassignon MJ. Refractive and topographic results of benzalkonium chloride-assisted transepithelial crosslinking. J Cataract Refract Surg. 2012; 38:1000-5. | Article | PubMed

21. Stojanovic A, Chen X, Jin N, Zhang T, Stojanovic F, Raeder S and Utheim TP. Safety and efficacy of epithelium-on corneal collagen cross-linking using a multifactorial approach to achieve proper stromal riboflavin saturation. J Ophthalmol. 2012; 2012:498435. | Article | PubMed
Abstract | PubMed FullText

22. Alhamad TA, O'Brart DP, O'Brart NA and Meek KM. Evaluation of transepithelial stromal riboflavin absorption with enhanced riboflavin solution using spectrophotometry. J Cataract Refract Surg. 2012; 38:884-9. | Article | PubMed

\section{Citation:}

Badawi AE. Visual and topographic impacts of trans-epithelial versus epithelium-off corneal collagen cross-linking in adult keratoconus. J Eye Ophthalmol. 2016; 3:1. http://dx.doi.org/10.7243/2055-2408-3-1 